Revue internationale P.M.E.

Économie et gestion de la petite et moyenne entreprise

Revue

internationale

PME

\title{
Prendre les rênes de la PME familiale et se faire accepter
}

\section{Vivi Koffi et Jean Lorrain}

Volume 24, numéro 2, 2011

URI : https://id.erudit.org/iderudit/1012686ar

DOI : https://doi.org/10.7202/1012686ar

Aller au sommaire du numéro

Éditeur(s)

Presses de l’Université du Québec

ISSN

0776-5436 (imprimé)

1918-9699 (numérique)

Découvrir la revue

Citer cet article

Koffi, V. \& Lorrain, J. (2011). Prendre les rênes de la PME familiale et se faire accepter. Revue internationale P.M.E., 24(2), 81-110.

https://doi.org/10.7202/1012686ar

\section{Résumé de l'article}

Cette étude de cas comparative présente comment les prédécesseurs hommes et femmes amènent leurs successeurs à se faire accepter par les membres de leur entreprise. Pour amener ceux-ci à l'acceptation, les prédécesseures adoptent des comportements différents de ceux des prédécesseurs. Faisant confiance aux successeurs, elles les protègent, les habilitent, adoptent un style de leadership « maternaliste » et mettent l'accent sur le travail collaboratif. Pour leur part, les prédécesseurs inspirent les successeurs, les habilitent, les amènent à faire leurs preuves et mettent l'accent sur des rencontres dyadiques. De plus, le successeur, pour être accepté, doit se faire intégrer en douceur, faire montre des valeurs et comportements compatibles avec ceux de l'entreprise, manifester intérêt, initiative, engagement, investissement de temps et d'énergie et gagner confiance et respect. Le processus d'acceptation vécu par les successeurs pour se faire accepter par le personnel de l'entreprise diffère donc selon qu'on est homme ou femme prédécesseur. Cette recherche montre l'importance de tenir compte du sexe du prédécesseur dans les études sur la succession dans les PME familiales.
Ce document est protégé par la loi sur le droit d'auteur. L'utilisation des services d’Érudit (y compris la reproduction) est assujettie à sa politique d'utilisation que vous pouvez consulter en ligne.

https://apropos.erudit.org/fr/usagers/politique-dutilisation/ 


\title{
Prendre les rênes de la PME familiale et se faire accepter
}

\author{
Vivi KOFFI \\ Université de Moncton
}

Jean LORRAIN

Université du Québec à Trois-Rivières

\section{MOTS CLÉS}

\section{Succession - Relève - PME familiale - Transfert de direction - Genre - Leadership}

\begin{abstract}
LES AUTEURS
VIVI KOFFI est professeure agrégée au Département d'administration de la Faculté d'administration de l'Université de Moncton où elle est aussi directrice adjointe du département. Elle y enseigne le management et le comportement organisationnel. Ses champs d'intérêt sont l'entrepreneuriat féminin, la transmission des entreprises et le genre et leadership des dirigeants. Courriel: <vivi.koffi@umoncton.ca>.

JEAN LORRAIN, professeur retraité au Département des sciences de la gestion à l'Université du Québec à Trois-Rivières, a mené une multitude de travaux de recherche dans le domaine de la psychologie entrepreneuriale au cours de sa carrière. Les résultats de ces travaux ont mené à de multiples communications lors de congrès scientifiques, dont ceux de Babson College, et à de nombreux articles, et ce, dans des revues internationales. Courriel: <jean. lorrain@uqtr.ca>.
\end{abstract}

\section{RÉSUMÉ}

Cette étude de cas comparative présente comment les prédécesseurs hommes et femmes amènent leurs successeurs à se faire accepter par les membres de leur entreprise. Pour amener ceux-ci à l'acceptation, les prédécesseures adoptent des comportements différents de ceux des prédécesseurs. Faisant confiance aux successeurs, elles les protègent, les habilitent, adoptent un style de leadership «maternaliste» et mettent l'accent sur le travail collaboratif. Pour leur part, les prédécesseurs inspirent les successeurs, les habilitent, les amènent à faire leurs preuves et mettent l'accent sur des rencontres dyadiques. De plus, le successeur, pour être accepté, doit se faire intégrer en douceur, faire montre des valeurs et comportements compatibles avec ceux de l'entreprise, manifester intérêt, 
initiative, engagement, investissement de temps et d'énergie et gagner confiance et respect. Le processus d'acceptation vécu par les successeurs pour se faire accepter par le personnel de l'entreprise diffère donc selon qu'on est homme ou femme prédécesseur. Cette recherche montre l'importance de tenir compte du sexe du prédécesseur dans les études sur la succession dans les PME familiales.

\section{ABSTRACT}

This comparative case study puts the emphasis on the ways used by men-and women-predecessors to be accepted by the other members of their enterprise. To bring the latter to the acceptation of the successor, the women-predecessors adopt different behaviors than the men-predecessors. Giving their confidence to the successor, the women-predecessors protect them, help them, adopt a "maternal » style of leadership, and put the emphasis on collaborative work. As for the predecessors, they inspire the successors, help them, bring them to prove their ability, and organize dyadic meetings. Furthermore, in order to be accepted, the successor must be integrated slowly within the enterprise, show values and behaviors compatible with those of the enterprise, show interest, initiative, involvement, investment of time and energy, and gain confidence and respect. The acceptation process experienced by the successors to be accepted by the other members of their enterprise is therefore different according whether we are a man- or a woman-predecessor. This research shows all the importance to take into account the gender of the predecessor in the studies related to the succession within family SMBs (Small and Medium Businesses).

\section{RESUMEN}

Este estudio de caso comparativo muestra como los predecesores-hombres y mujeres- impulsan a sus sucesores a ser aceptados por los integrantes de sus empresas. Para conducirlos a la aceptación, las predecesoras adoptan comportamientos distintos a los de los predecesores. Al otorgar su confianza a los sucesores, protegiéndolos y habilitándolos, ellas adoptan un estilo de liderazgo "maternal», enfatizando el trabajo colaborativo. Los predecesores, por su parte, inspiran a los sucesores, los habilitan, los conducen a dar prueba de sus aptitudes y ponen énfasis en los encuentros diádicos. Además, a fin de ser aceptado, el sucesor debe dejarse integrar lentamente, mostrar que posee valores compatibles con los de la empresa, y también manifestar interés, iniciativa, compromiso, inversión de tiempo y energía, así como capacidad de ganarse la confianza y el respeto. Por tanto, el proceso de aceptación vivido por los sucesores para lograr la aceptación del personal de la empresa difiere, dependiendo de si el predecesor es hombre o mujer. La investigación muestra la importancia de tener en cuenta el sexo del predecesor en los estudios sobre la sucesión en las PyMEs familiares.

\section{ZUSAMMENFASSUNG}

Diese vergleichende Fallstudie zeigt auf, wie weibliche und männliche Unternehmer vorgehen, damit ihre Nachfolger von den bestehenden Mitarbeitern akzeptiert werden. Frauen und Männer unterscheiden sich diesbezüglich. Frauen vertrauen 
ihren Nachfolgern, beschützen, befähigen, pflegen einen matriarchalischen Führungsstil und bevorzugen einen Arbeitsstil geprägt von Zusammenarbeit. Männer inspirieren ihre Nachfolger, befähigen sie ebenfalls, ermöglichen ihnen die Sporen abzuverdienen und arbeiten vor allem in Zweierteams. Der Nachfolger seinerseits muss, um von den Mitarbeitenden akzeptiert zu werden, mit dem Unternehmen kompatible Werte und Verhalten manifestieren, Interesse zeigen, Initiative und Engagement übernehmen, Zeit und Energie investieren um Respekt und Vertrauen aufzubauen. Der Aufnahmeprozess läuft demnach unterschiedlich ab, je nachdem ob der Vorgänger eine Frau oder ein Mann ist. Diese wichtige Komponente ist bei der Forschung von Nachfolgeprozessen in Familien-KMU zu berücksichtigen.

\section{Introduction}

Malgré l'importance capitale que revêtent les PME familiales dans le développement économique, social et culturel des nations (Astrachan et Shanker, 2003 ; Cadieux et Brouard, 2009), une problématique de plus en plus d'actualité préoccupe les propriétaires-dirigeants de ces entreprises. Que ce soit au Québec, au Canada, aux États-Unis et dans les autres pays industrialisés, la plupart des dirigeants, près d'un sur deux, ont plus de 50 ans et sont aux prises avec la dynamique de la transmission familiale, c'est-à-dire la transmission de leur entreprise à un successeur, membre de la famille (Cadieux et Brouard, 2009; Sharma, 2004). Par exemple, selon les résultats de l'étude de Bruce et Picard (2005), sur les chefs d'entreprises canadiennes, $41 \%$ vont transférer d'ici les cinq prochaines années et $71 \%$ dans les dix années à venir, mais seulement $35 \%$ disent avoir un plan pour vendre ou transférer. De plus, la transmission familiale, un phénomène en régression (Cadieux et Brouard, 2009), serait un processus long et complexe dont la réussite reposerait sur bon nombre de facteurs. L'un des facteurs serait l'acceptation du successeur comme futur chef d'entreprise par les ressources humaines impliquées dans l'entreprise (Barach, Ganitsky, Carson et Doochin, 1988; Bayad et Barbot, 2002; Fiegener, Brown, Prince et File, 1996). Or, ces auteurs précités n'ont pas pu, à partir de leurs études, expliquer de façon empirique la manière dont les chefs d'entreprises (prédécesseurs) s'y prennent pour faire accepter leur progéniture (successeurs) par les membres de l'entreprise.

De plus, malgré la multitude des travaux réalisés sur la problématique de la transmission familiale au cours des trois dernières décennies, très peu de chercheurs dans ce domaine se sont intéressés, à ce jour, au processus successoral lorsque le prédécesseur était une femme et encore moins, à la façon dont celle-ci s'y prenait pour passer le relais (Barrett et Moores, 2009; Cadieux, Lorrain et Hugron, 2002; Richer, St-Cyr et Youssef, 2004; Salganicoff, 1990; Sharma, 2004; Sonfield et Lussier, 2009); pourtant, au Canada, plus de $40 \%$ des entrepreneurs sont des femmes (Richer et St-Cyr, 2007). De plus, l'ensemble des travaux réalisés en entrepreneuriat 
féminin sur le mode de direction des femmes, depuis l'étude de Hisrich et Brush en 1984 jusqu'à celle plus récente de Buttner (2001), montre pour la plupart, selon la perspective du féminisme social, que les comportements des femmes chefs d'entreprise à l'égard de leur personnel et de leur entourage d'affaires diffèrent des comportements adoptés par leurs homologues masculins et seraient, selon toute vraisemblance, plus relationnels et plus efficaces (Buttner, 2001; Chodorow, 1978; Fondas, 1997; Gilligan, 1986; Miller, 1991; Rapoport et Bailyn,1996; Weisinger, 1998). La spécificité des comportements des entrepreneurs hommes et femmes lors du transfert des entreprises familiales et l'urgence d'appuyer ses prédécesseurs dans une succession réussie donnent toute sa pertinence à l'objectif principal de cette recherche. Cet objectif est donc d'arriver à comprendre et à décrire comment les femmes et les hommes chefs d'entreprises familiales s'y prennent pour amener leur successeur à se faire accepter par les employés avec qui l'entreprise a été bâtie, à partir du moment où l'acceptation semble être un gage de la pérennité de l'entreprise (Barach et al., 1988; Sathe, 1985). En d'autres termes, quels seraient les comportements gagnants que les prédécesseurs hommes et femmes adopteraient pour assurer l'acceptation de leurs successeurs? Et lorsqu'on parle de successeur accepté, qu'est-ce qui le caractérise?

Afin de répondre à ces questions, notre démarche consistera à présenter, premièrement, le cadre d'analyse exposant les enjeux de la problématique de succession d'entreprise familiale. Nous y tiendrons compte dans un premier temps des particularités du style de gestion des femmes, des écrits sur la transmission des entreprises familiales dans un contexte de socialisation d'un nouvel employé et des travaux sur le concept d'acceptation du successeur. Dans un deuxième temps, nous présenterons la démarche méthodologique utilisée, suivie des résultats obtenus; ces derniers seront discutés avant de conclure ce travail.

\section{Recension des écrits}

\subsection{Le mode relationnel des femmes chefs d'entreprise}

Le leadership est un construit dont la définition n'a pas trouvé consensus jusqu'à ce jour auprès des auteurs qui s'y sont intéressés. De façon générale, il est perçu comme l'aptitude d'une personne visionnaire à adopter des comportements d'influence, de motivation et de mobilisation d'autres individus et à leur permettre de contribuer à l'efficacité et à la réussite de leur organisation (Alimo-Metcalfe, 2007; Bass et Avolio, 1994). Autrement dit, parler de leadership des prédécesseurs dans le processus d'acceptation des successeurs 
dans notre étude, c'est faire référence aux comportements adoptés par les prédécesseurs à l'endroit des successeurs en interaction avec les membres de l'entreprise. Or, il semblerait selon certaines approches théoriques, même si la question n'est pas tranchée, que les comportements adoptés par les femmes et par les hommes en situation de direction seraient les mêmes pour certains, complémentaires et différents pour d'autres. Cette confusion nous amène à nous poser la question de savoir quels comportements les prédécesseures et prédécesseurs adoptent dans le processus d'acceptation du successeur.

Justement, l'approche du féminisme libéral mentionne qu'en matière de leadership, les femmes et les hommes sont égaux à tous égards. Même si des différences psychologiques peuvent exister, étant dotées de rationalité, les femmes sont porteuses du même potentiel que les hommes, donc munies des mêmes capacités de leadership. Les différences que l'on peut remarquer par rapport à leurs réalisations et performances en situation de gestion sont dues au fait que les femmes n'ont pas atteint leur plein potentiel ou sont victimes de discrimination dans leur environnement (Ahl, 2006; Fischer, Reuber et Dyke, 1993; Hollander, 1992). Il n'y a donc pas de raison pour que les femmes soient moins efficaces que les hommes.

Pour la théorie postructuraliste qui s'apparente à l'approche systémique, la question n'est pas de savoir qui sont les hommes et les femmes, mais comment la féminité et la masculinité sont construites dans les organisations. Le genre est un phénomène socialement construit, accompli ou performé autour des mécanismes de séparation et de hiérarchisation, qui varie d'un milieu à un autre (Ahl, 2006; Bruin, Brush et Welter, 2007; Butler, 1990; Ely et Padavich, 2007).

Le féminisme social, radical ou psychanalytique (Ahl, 2006), approche peu développée sur le plan théorique, mentionne que les différences essentielles existent entre les hommes et les femmes et ces différences sont à l'avantage des femmes surtout dans la perspective sociale des économies avancées. Pour Duchéneaut (1997), les tenants de cette approche s'appuient sur des résultats d'études empiriques auprès des femmes entrepreneures ayant réussi. On peut citer par exemple les travaux d'Helgesen (1990) qui indiquent des différences dans la manière dite féminine de faire les choses, différences, à l'avantage des femmes, lorsqu'on tient compte des caractéristiques essentielles dont le dirigeant doit faire montre tels que le rythme de travail, l'intelligence émotionnelle, les compétences interpersonnelles et communicationnelles (Chodorow, 1978; Gilligan, 1986; Miller, 1986). C'est dans cette optique que certains auteurs (Chodorow, 1978; Buttner, 2001; Fletcher, 1998; Gilligan, 1986; Miller, 1986) ont élaboré la théorie 
relationnelle et que d'autres (Alimo-Metcalfe, 2007; Avolio et Bass, 2002; Eagly, Johannesen-Schmidt et Van Engen, 2003; Foschi, 2000) en sont arrivés à la théorie du leadership transactionnel et transformationnel.

Cet article s'appuie donc sur la théorie relationnelle et la théorie du leadership transformationnel afin d'atteindre son objectif, celui de comprendre les comportements adoptés par les chefs d'entreprises dans le processus d'acceptation de leurs successeurs.

\subsubsection{La théorie relationnelle}

La théorie relationnelle mentionne que les femmes sont différentes des hommes et que cette différence est à leur avantage, car elles auraient un sens intériorisé de connexion aux autres, leur permettant de se mettre en relation. Elles adopteraient donc une approche plus personnalisée avec leurs employés et leurs clients, mettant ainsi l'accent sur la croissance et le développement de l'individu qui surviendraient mieux dans un contexte de relations où il y a interaction (Buttner, 2001; Fletcher, 1998; Gilligan, 1986; Miller, 1986).

Ces interactions comportent quatre dimensions : la protection, l'habilitation mutuelle, l'accomplissement de soi et la création d'équipe de travail. a) Protéger signifie que les femmes épaulent, secourent et portent assistance. b) L'habilitation mutuelle leur permet de rendre les employés capables d'accomplir et de contribuer à la réalisation des activités de l'entreprise. c) L'accomplissement renvoie à l'usage des habiletés relationnelles pour améliorer leur propre croissance professionnelle et leur efficacité au travail. d) La création d'équipe a trait au fait de créer les conditions de base dans lesquelles la vie de groupe peut s'épanouir. Tsui, Pearce, Porter et Tripoli (1997) ont trouvé, comme Rapoport et Bailyn (1996), Fondas (1997) et Weisinger (1998), que certaines organisations qui favorisent les pratiques de type relationnel sont très efficaces, ont un taux d'absentéisme et de roulement du personnel très bas et un indice d'honnêteté très élevé. Les résultats de l'étude de cas (Buttner, 2001) ayant porté sur le comportement de leadership de 90 femmes chefs d'entreprises indiquent que ces dernières ont mis l'accent, dans l'exercice de leur fonction de direction, sur la protection des employés, sur leur bienveillance, leur accomplissement et sur le travail d'équipe. Elles ont aussi défini le pouvoir en termes de capacité à s'occuper des autres et à contribuer à leur bien-être. Malgré ces résultats, Buttner (2001) fait une mise en garde: même si cette théorie peut servir de cadre de référence pour mieux comprendre le comportement des femmes chefs d'entreprises, elle n'a pas la prétention de parler pour toutes les femmes et ne tend pas à affirmer 
que seules les femmes y souscrivent, laissant ainsi la place aux hommes chefs d'entreprises, acception qui rejoint la théorie du leadership transactionnel et transformationnel (Avolio et Bass, 2002; Brush, 1992; Rosener, 1990).

\subsubsection{Leadership transactionnel et transformationnel}

Plusieurs auteurs se sont servis de la théorie du leadership transactionnel et transformationnel (Alimo-Metcalfe, 2007; Avolio et Bass, 2002; Eagly et al., 2003; Foschi, 2000), pour comprendre la différence entre le leadership des femmes et des hommes.

Le leader transactionnel fournit à ses subordonnés des récompenses contingentes, en échange d'objectifs imposés. Ce type de leadership implique un style de management de clarification des responsabilités du subordonné pour atteindre les objectifs, et de sanctions, dans le cas où les objectifs ne sont pas atteints (Bass et Avolio, 1994). On rencontre quatre comportements possibles du leadership transactionnel: $a$ ) l'attribution de renforcements en fonction du rendement, $b$ ) la gestion par exception active, $c$ ) la gestion par exception passive et $d$ ) le laisser-faire, qui est marqué par un laisser-aller général au regard des responsabilités.

Les dirigeants transformationnels, quant à eux, sont charismatiques, persuasifs et capables de stimuler intellectuellement les autres. Tout leader de ce type est en mesure de «transformer» les membres de son organisation en les rendant plus conscients de l'importance de leurs tâches et en les aidant à étendre leur horizon au-delà de leurs intérêts personnels pour mener à bien la mission de l'entreprise. Quatre caractéristiques définissent le leadership transformationnel: $a$ ) le charisme, où le leader est porteur d'une vision, b) l'inspiration, où il insuffle des attentes élevées par rapport à la passion et à la vision, $c$ ) la stimulation intellectuelle, où il permet l'acquisition de compétences nécessaires et $d$ ) la bienveillance, où il manifeste de la bonté et de l'empathie. Cependant, comment cela se produit-il dans un contexte de direction?

Selon certains auteurs, les hommes sont en général plus portés à utiliser les aspects du style transactionnel, c'est-à-dire les récompenses et les sanctions, le management par exception passif, par exception actif et le style laisser-faire dans des situations de gestion, que les aspects du style transformationnel plus souvent utilisé par les femmes gestionnaires (Alimo-Metcalfe, 2007; Avolio et Bass, 2002; Eagly et al., 2003; Koffi, 2008; Rosener, 1990). Brush (1992), Buttner (2001) et Robinson (2001) mentionnent que les femmes entrepreneures adoptent un style plus participatif et visent à intégrer travail et famille, alors que les hommes privilégient l'esprit d'entreprise et ont 
tendance à préférer jouer un rôle de supervision plutôt que de collaboration. L'étude de Riebe (2005), réalisée auprès de 27 chefs d'entreprises à succès, indique comme Buttner (2001), Koffi et Lorrain (2011) que, pour réussir, ces entrepreneures interviewées ont déclaré adopter des comportements relatifs à la collégialité et à la démocratie.

Toutefois, alors que certains chercheurs notent une différence entre les styles de leadership des hommes et des femmes, d'autres sont arrivés à la conclusion que le sexe des gestionnaires n'influence pas leur style de leadership (Cole, 1997; Powell, 1990; Vikinias et Cartan, 1993; Van Engen, Van Der Leeden et Willemsen, 2001; Vecchio, 2003). Devant cette absence de consensus, ces études, pour la plupart conceptuelles, nous amènent à vouloir comprendre les types de comportements adoptés selon qu'on soit homme ou femme dans un contexte de transmission des entreprises.

\subsection{La transmission des entreprises familiales: processus de socialisation du successeur}

Selon Cadieux et Brouard (2009), on parle de transmission lorsqu'une entreprise assure sa continuité par la mise en place effective de un ou plusieurs successeurs ou d'un repreneur et par le retrait du prédécesseur ou du cédant. On en compte trois types: la transmission familiale, où le propriétaire (prédécesseur) transmet l'entreprise à au moins un membre de la famille; la transmission interne, par laquelle le propriétaire ou cédant transmet l'entreprise à un ou plusieurs membres du personnel de l'entreprise qui n'ont aucun lien de parenté avec lui; et la transmission externe, qui survient lorsque, faute de trouver un membre de la famille ou du personnel, le propriétaire cédant se tourne vers un repreneur externe. Notre étude se concentre sur la transmission familiale qui, appelée aussi succession, est un processus comportant souvent deux dimensions: le transfert de direction et le transfert de propriété (Hugron, 1993). Le transfert de direction se produit dans un processus dynamique de socialisation organisationnelle du successeur au cours duquel les rôles et les fonctions du prédécesseur et du successeur, membre de la famille, évoluent de manière imbriquée (Handler, 1990), en vue de transférer au successeur la philosophie de vie, la philosophie de gestion, les responsabilités, les attitudes, l'autorité, le pouvoir et le leadership (Cabrera-Suárez, 2005; Hugron, 1993; Lajeunesse, 1989).

\subsubsection{La socialisation «repreneuriale» du successeur}

Il est évident que le successeur, qu'il acquière son savoir-faire dans le giron familial ou à l'externe, passe nécessairement par le processus de socialisation 
organisationnelle, une fois son entrée effectuée dans l'entreprise familiale (D'Andria et Chalus-Sauvannet; 2007; Koffi, 2008). La socialisation est un processus par lequel le nouvel arrivant apprend le système des valeurs, les normes, les comportements et les connaissances qui lui permettent d'être membre participant de l'organisation, de la société et du groupe qu'il vient de joindre (Ardts, Jansen et Van der Velde, 2001; Ashforth, Saks et Lee, 1998; Wanous, 1992). Processus d'appropriation de la culture organisationnelle dont l'un des objectifs est l'acceptation mutuelle des membres, la socialisation met l'accent sur l'implication de l'employé et de l'organisation (Wanous, 1992; Wanous et Reichers, 2000). Une grande partie de ce processus d'apprentissage commencerait avant l'entrée dans l'organisation du nouvel employé et se poursuivrait tout au cours de sa carrière, principalement durant trois des quatre phases du processus de succession: les phases d'initiation, d'intégration et de règne conjoint (Hugron, 1993; Jolicœur, 1994; Lajeunesse, 1989; Koffi 2008). Au cours de la phase d'initiation, le prédécesseur initie son successeur aux rouages de l'entreprise en suscitant son intérêt. Cette initiation se poursuit jusqu'à la phase dite d'intégration, où le prédécesseur procède à l'intégration sociale du successeur en lui permettant d'occuper des postes à temps partiel où l'on peut assister à un début du transfert du savoir, du savoir-être et du savoir-faire. À la phase du règne conjoint, l'héritier choisi, non seulement selon ses compétences et l'intérêt démontré (Barbot et Richome-Huet, 2007; Haddadj et D'Andria, 2001), mais aussi selon la confiance et, surtout, selon la qualité de la relation existant entre le prédécesseur et le successeur et tous les autres acteurs (Barach et al.; 1988; Lansberg et Astrachan, 1994), fait son entrée de façon plus officielle dans l'entreprise. Il s'y familiarise, se fait confirmer en travaillant avec les employés et le prédécesseur (Hugron, 1993; Lajeunesse, 1989). Ce dernier, jouant des rôles précis (Cadieux et al., 2002), lui transfère la philosophie de vie, la philosophie de gestion, les responsabilités et le pouvoir (Hugron, 1993; Lajeunesse, 1989) jusqu'à ce qu'il se retire complètement à la phase du désengagement. Cependant, selon Barach et al. (1988) ainsi que Sathe (1985), afin de pouvoir diriger l'entreprise dans la voie du succès et de la pérennité, il est important que le successeur se fasse accepter par les membres de l'entreprise. Qu'est-ce donc l'acceptation d'un successeur dans le cadre de la transmission des entreprises familiales? Comment cette acceptation se produit-elle auprès du prédécesseur et des employés qui ont vu évoluer le successeur et l'entreprise?

L'acceptation du successeur. Il est important de souligner que le concept d'acceptation du nouvel employé par les pairs n'a pas fait l'objet d'attention particulière de la part des chercheurs. Poudrier (2000) définit l'acceptation d'un employé par la congruence entre les objectifs organisationnels et ceux de la personne évaluée. Dans le même ordre d'idées, pour Longenecker 
et Nykodym (1996), étudiant le concept dans un contexte de supervision, l'acceptation du collaborateur augmente lorsque le superviseur et le collaborateur ont une perception et une compréhension communes des objectifs à atteindre. Murphy et Cleveland (1995) mentionnent, pour leur part, que l'accord sur l'accomplissement des tâches et la perception commune des normes de performance sont deux facteurs essentiels à l'acceptation.

Dans le contexte des entreprises familiales, les études importantes élaborées sur le concept d'acceptation du successeur sont celles de Sathe (1985) et de Barach et al. (1988). Selon Sathe (1985), la réussite du transfert managérial est déterminée par le degré d'acceptation du successeur. Cette acceptation surviendrait lorsque les membres de l'entreprise perçoivent les croyances, les valeurs et les comportements du successeur compatibles et conformes à la culture organisationnelle. Pour Barach et al. (1988), avant que le successeur ne puisse prétendre succéder au prédécesseur, ce dernier devrait s'assurer du niveau d'acceptation dont jouit sa relève dans l'organisation, acceptation qui n'est pas automatique.

Nous constatons que les études mentionnées ci-dessus ne nous indiquent pas de façon claire les dimensions de l'acceptation et les comportements adoptés par les prédécesseurs pour en favoriser le gain auprès du personnel de l'entreprise. De plus, elles nous informent moins sur la différenciation de ces comportements par le sexe. Il n'en demeure pas moins que les travaux axés sur le féminisme social, plus particulièrement sur la théorie relationnelle (Buttner, 2001) et sur la théorie du leadership transactionnel et transformationnel, nous permettent de supposer que la capacité des femmes à établir des liens avec les autres, leur propension à favoriser le développement de leurs employés, pourraient amener la prédécesseure à adopter des comportements différents du prédécesseur, comportements qui favoriseraient plus rapidement l'acceptation de son successeur. Comme postulé dans le modèle conceptuel (voir la figure 1) qui suit, les prédécesseurs hommes et femmes, chefs d'entreprises familiales en situation de retrait, adopteraient un mode comportemental axé sur les pratiques relationnelles (Buttner, 2001) et sur le leadership transformationnel et transactionnel (Bass et Avolio, 1994; Eagly et al.,2003) pour amener leurs successeurs à gagner l'acceptation auprès des employés de leurs entreprises. Dans le cas contraire, quels types de comportements adopteront-ils? La méthodologie de recherche présentée dans les lignes qui suivent nous permettra d'élucider ces questions. 
FIGURE 1

La relation entre les comportements des prédécesseurs et l'acceptation du successeur par les membres de l'entreprise

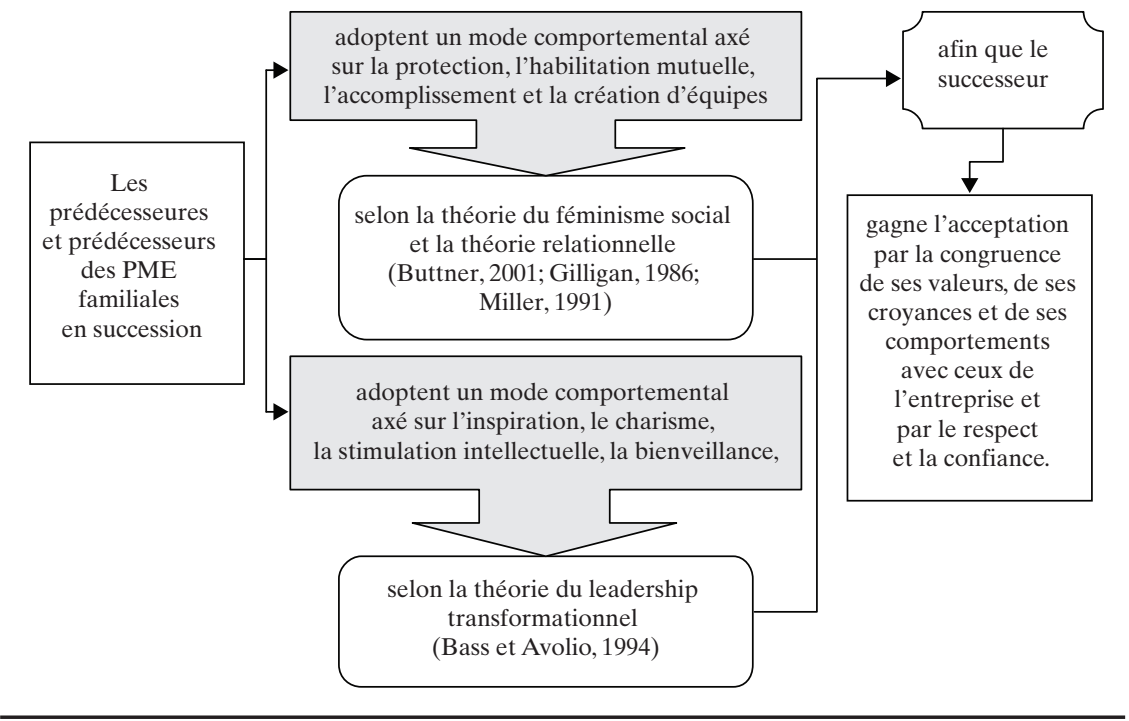

\section{Méthodologie}

\subsection{Constitution de l'échantillon}

Afin de comprendre les modes comportementaux adoptés par les prédécesseures et prédécesseurs pour amener leurs successeurs à gagner l'acceptation auprès des membres de l'entreprise, nous avons élaboré un design méthodologique dont les grandes lignes se présentent comme suit. Nous nous inscrivons dans une optique de recherche qualitative puisque la problématique est nouvelle. Pour ce faire, nous avons retenu la stratégie de l'étude de cas exploratoire et multisite (Hlady-Rispal, 2002) car, selon Yin (1994), cette stratégie est recommandée lorsque le phénomène à l'étude fait partie d'une problématique pour laquelle il n'existe que peu d'études empiriques (Grenier et Josserand, 1999). Nous avons opté pour une procédure d'échantillonnage théorique, à savoir que les sept cas sont choisis pour des motifs non statistiques, mais théoriques (Hlady-Rispal, 2002). Nous avons tenu compte des critères d'échantillonnage suivants: le processus de succession de la première à la deuxième génération entamé et possiblement terminé; la succession mère-fille ou fils et père-fille ou fils ; une entreprise du secteur manufacturier ou du secteur des services comptant de 10 à 250 employés; l'employé retenu 
doit avoir travaillé dans l'entreprise avec le propriétaire-dirigeant avant ou pendant que le successeur fait son entrée dans l'entreprise. De plus, la rareté des femmes chefs d'entreprises en situation de transfert managérial nous a amenés à opter pour un échantillonnage par choix raisonné, c'est-à-dire que nous avons choisi délibérément les sujets, au jugement, selon qu'ils ont été trouvés aptes à autoriser la compréhension et l'interprétation des comportements de leadership qu'ils adoptent pour faire accepter leur progéniture. Compte tenu des critères retenus, sept entreprises (quatre prédécesseures et trois prédécesseurs) dans le processus du premier transfert générationnel et dans lesquelles au moins un membre de la famille détient le contrôle de la propriété et de la gestion (Cadieux et al., 2002), ont été retenues (voir le tableau 1). Deux prédécesseures ont déjà transféré l'entreprise à leurs successeurs. Les cinq autres ayant exprimé leur intention ferme de transmettre l'entreprise sont à un stade avancé du processus. Le nombre d'entretiens effectués, les profils des PME féminines et masculines retenues en tenant compte de leur date de fondation, des secteurs d'activité (deux manufacturières et quatre du secteur des services), du nombre d'employés (de 15 à 150), du sexe des successeurs (trois successeures et quatre successeurs), du chiffre d'affaires (de deux à sept millions), de l'état du transfert et du pourcentage d'actions détenues de part et d'autre, sont présentés dans le tableau 1.

\subsection{Collecte et analyse des données}

Premièrement, la collecte des données a été réalisée de février 2004 à avril 2006. Les données présentées ont été obtenues grâce à des entretiens individuels semi-directifs (27 au total) d'une durée moyenne d'une heure et demie. Les trois guides d'entretiens basés sur les éléments du cadre conceptuel se structuraient autour des rubriques suivantes: la présentation de l'entreprise, le processus de succession, les comportements de leadership et l'acceptation du successeur. En vue de la triangulation des données ${ }^{1}$, ces entrevues ont eu lieu avec sept prédécesseurs, sept successeurs et treize employés.

1. La triangulation des données permet de confronter les données recueillies des différentes sources (dans ce cas-ci, la prédécesseure, le successeur, les deux employés et les documents obtenus de chaque entreprise) afin d'avoir des résultats plus robustes. 


\section{TABLEAU 1}

\section{Profil des entreprises des femmes et des hommes prédécesseurs}

\begin{tabular}{|c|c|c|c|c|}
\hline $\begin{array}{c}\text { Femmes } \\
\text { prédécesseures }\end{array}$ & Nature & Montage & Terroir & Binette \\
\hline $\begin{array}{l}\text { Date de } \\
\text { fondation }\end{array}$ & 1987 & 1988 & 1991 & 1986 \\
\hline $\begin{array}{l}\text { Secteur } \\
\text { d'activité }\end{array}$ & Services & Services & Manufacturier & Services \\
\hline $\begin{array}{l}\text { Nombre } \\
\text { d'employés }\end{array}$ & 59 & 50 à 150 & 32 & 26 \\
\hline Successeur & Fille & Fils & Fils & Fils \\
\hline $\begin{array}{l}\text { Chiffre } \\
\text { d'affaires }\end{array}$ & 3 à 4 millions & 2 à 3 millions & 3 millions & 6 à 7 millions \\
\hline $\begin{array}{l}\text { État du } \\
\text { transfert }\end{array}$ & Terminé & Règne conjoint & Terminé & En processus \\
\hline Actions & $\begin{array}{l}\text { Prédécesseure: } \\
0 \% \\
\text { Successeure: } \\
33 \% \\
\text { Actionnaires: } \\
66 \%\end{array}$ & $\begin{array}{l}\text { Prédécesseure: } \\
33 \% \\
\text { Successeur: } \\
0 \% \\
\text { Actionnaires: } \\
66 \%\end{array}$ & $\begin{array}{l}\text { Prédécesseure: } \\
52 \% \\
\text { Successeur: } 48 \%\end{array}$ & $\begin{array}{l}\text { Prédécesseure: } \\
50 \% \\
\text { Successeurs: } \\
\text { fils, } 25 \% \text {; } \\
\text { fille, } 25 \% \text {. }\end{array}$ \\
\hline Entretiens & 3 & 4 & 4 & 4 \\
\hline $\begin{array}{c}\text { Hommes } \\
\text { prédécesseurs }\end{array}$ & Cérami & Sanite & Jetpur & \\
\hline $\begin{array}{l}\text { Date de } \\
\text { fondation }\end{array}$ & 1975 & 1980 & 1999 & \\
\hline $\begin{array}{l}\text { Secteur } \\
\text { d'activité }\end{array}$ & $\begin{array}{l}\text { Commerce de } \\
\text { détail }\end{array}$ & Services & Manufacturier & \\
\hline $\begin{array}{l}\text { Nombre } \\
\text { d'employés }\end{array}$ & 15 & 20 & 46 & \\
\hline Successeur & Fille & Fils & Fille & \\
\hline $\begin{array}{l}\text { Chiffre } \\
\text { d'affaires }\end{array}$ & 2 millions & 6 millions & 5 millions & \\
\hline $\begin{array}{l}\text { État du } \\
\text { transfert }\end{array}$ & Presque terminé & En processus & En processus & \\
\hline Actions & $\begin{array}{l}\text { Prédécesseur: } \\
100 \%\end{array}$ & $\begin{array}{l}\text { Prédécesseur: } \\
100 \%\end{array}$ & $\begin{array}{l}\text { Prédécesseur: } \\
63 \% \\
\text { Successeur: } 6 \% \\
3 \text { employés: } 31 \% \\
\end{array}$ & \\
\hline Entretiens & 4 & 4 & 4 & \\
\hline
\end{tabular}


Deuxièmement, les entretiens individuels au nombre de 27 ont été enregistrés et successivement retranscrits aussitôt, fournissant ainsi une première analyse qui nous a permis de nous ajuster lors des entretiens subséquents (Yin, 1994). Ensuite, sept cas d'entreprises ont été rédigés. Le logiciel QSR NVivo 2.0 a été retenu pour l'analyse thématique des interviews ${ }^{2}$ (Paillé et Mucchielli, 2003). Le principe d'analyse à la base du logiciel NVivo 2.0 relève d'une démarche de décontextualisation-recontextualisation du corpus (Paillé et Mucchielli, 2003). Cette décontextualisation consiste à sortir de son contexte un extrait du discours afin de le rendre sémantiquement indépendant dans le but de créer des catégories ou des thèmes regroupant tous les extraits d'un sujet en particulier (Deschenaux et Bourdon, 2005). Tout d'abord, la flexibilité de l'analyse thématique nous a permis de prédéfinir et de hiérarchiser les divers thèmes ou concepts du cadre conceptuel (voir la figure 1) qui portent sur le processus de succession (Hugron, 1993; Lajeunesse, 1989), sur les quatre dimensions de la théorie relationnelle (Buttner, 2001) et les quatre dimensions du leadership transformationnel (Bass et Avolio, 1994) ainsi que sur les dimensions du concept d'acceptation (Barach et al., 1988). Nous avons aussi, par le fait même, ajouté les thèmes qui ont émergé au cours de l'analyse faite avec le logiciel NVivo 2.0. Pour la validation de ces thèmes, nous nous sommes basés sur leur récurrence, leur constance et l'importance qu'ils revêtent pour les répondants dans le corpus. Pour satisfaire à l'exigence de corroboration des données (Yin, 1994), nous avons confronté les différents points de vue exprimés par les répondants sur les différents sujets, aussi avons-nous tenu compte, dans la mesure du possible, des discours de quatre répondants dans chaque entreprise.

\section{Résultats}

Comment la prédécesseure et le prédécesseur qui décident, à la fin de leur carrière respective, de se retirer et de céder leurs entreprises à leur successeur, arrivent-ils à faire accepter ce dernier dans l'entreprise qu'ils ont créée et bâtie de leurs propres mains? Telle était la question que nous nous sommes posée. Tenter d'y répondre nous amène à l'analyse du discours des prédécesseurs. Dans une perspective comparative, nous décrirons d'abord les comportements de leadership selon qu'on est homme ou femme prédécesseur

2. L'analyse thématique permet de procéder systématiquement $a$ ) au repérage, $b$ ) au regroupement et $c$ ) subsidiairement à l'examen discursif des thèmes abordés dans notre corpus en reconstruisant une nouvelle structure avec les extraits thématisés ou codés dont nous nous sommes servis comme support aux résultats finaux. Nous avons spécifiquement opté pour la thématisation séquencée qui, selon Paillé et Mucchielli (2003), permet une analyse fine et uniforme du corpus. 
pour l'acceptation du successeur. Ensuite, nous déterminerons s'il y a une différence entre ces comportements lesquels, thématisés sous forme de stratégies de leadership, seront mis en relation avec les dimensions thématisées de l'acceptation.

\subsection{Analyse comparative: différences sexuées de styles d'accompagnement}

L'analyse comparative des comportements découlant des différents styles d'accompagnement des prédécesseures et prédécesseurs nous indique plus de différences que de similitudes. Le seul comportement adopté par les femmes et les hommes pour faire accepter leurs successeurs serait l'habilitation de ces derniers. Autrement dit, afin de favoriser l'acceptation de leurs successeurs, les quatre femmes chefs d'entreprises se distinguent de leurs homologues masculins en mettant l'accent sur la confiance, la protection, le leadership «maternaliste» et le travail collaboratif. Les hommes, de leur côté, tout en contrôlant leurs successeurs, les auraient amenés à faire des preuves, auraient été leur source d'inspiration et auraient mis l'accent sur le travail dyadique. Dans les lignes qui suivent, nous présenterons d'abord le comportement commun aux femmes et aux hommes, à savoir l'habilitation. Ensuite, il sera question successivement des comportements distinctifs des femmes et des hommes. Enfin, nous présenterons une description des dimensions de l'acceptation selon les répondants.

\subsubsection{Habiliter le successeur selon les femmes et les hommes chefs d'entreprise}

En habilitant les successeurs, les prédécesseurs hommes et femmes se sont appliqués à les rendre capables d'atteindre les objectifs relatifs aux responsabilités qui leur ont été confiées. Ils ont mis l'accent sur la formation à l'interne et à l'externe. Même si habiliter le successeur est important pour les deux sexes, ils n'ont pas procédé de la même façon. Dans le cadre de la formation des successeurs au sein de l'entreprise, les quatre femmes se sont appuyées sur l'expertise des consultants, des amis et des employés compagnons de travail. Elles ont aussi pratiqué un enseignement d'ouverture basé sur l'empathie, aiguisant ainsi la capacité des successeurs d'être à la fois experts et apprenants capables de remise en question. Voici ce que disent les répondants: «Je l'intégrais tranquillement, je la présentais à mes fournisseurs, aux gens d'expérience, à tout le monde et je voyais comment [...], ils l'acceptaient» (E1F-Int-Préd, $\left.62^{3}\right)$.

3. Première entreprise des femmes, interview de la prédécesseure, discours numéro 62. 
«Donc, dès l'achat, on a entrepris l'implantation des normes HACCP [...] et j'ai été mandaté de faire tout ça dans l'usine avec l'aide d'un consultant [...] C'est bon pour l'acceptation» (E3F-Int-Succ, $\left.24^{4}\right)$.

Les hommes, de leur côté, ont trouvé important de faire acquérir les différents savoirs à leurs successeurs par la formation à l'externe et à l'interne, et par l'expérience à l'interne acquise par le biais des projets spéciaux. Afin que les transferts des savoirs soient concrétisés, souvent, sur le plan technique, contrairement aux femmes qui privilégiaient l'apport des consultants, les prédécesseurs ont mis l'accent sur l'apprentissage tacite au sein de l'entreprise. C'est par cette approche pédagogique que la «progéniture» apprend tout en regardant comment le mentor, dans la majorité des cas, le prédécesseur, ou parfois les collaborateurs des prédécesseurs, procèdent concrètement. Cette façon de fonctionner aurait facilité l'acceptation selon leurs dires: «Caroline a eu deux coachs, elle a eu son père qui a fondé plusieurs compagnies, et qui, présentement, fait partie des chefs d'entreprise; elle a eu le contrôleur au point de vue administratif. [...] Aux yeux des autres, ça donne l'acceptation » (E3H-Int-Préd, $81^{5}$ ). Les prédécesseurs ont aussi privilégié le compagnonnage dont la finalité est d'amener l'employé à assurer la formation à l'interne du successeur, comme cela s'est produit dans le cas de $\mathrm{S}_{2} \mathrm{H}^{6}$ et $\mathrm{S} 3 \mathrm{H}:$ : Je le coachais un peu dans diverses tâches» (E2H-Int-Empl-B, 887).

\subsubsection{Quels comportements pour les femmes chefs d'entreprises?}

Faire confiance au successeur. La facilité à faire confiance distingue nettement les femmes des hommes. Hormis les bonnes relations entretenues pour la plupart avec les successeurs, sur la base de la confiance, les prédécesseures ont d'abord fait le choix du successeur qui, de son côté, a manifesté clairement le désir d'intégrer l'entreprise familiale et d'en prendre les rênes. Ensuite, elles se sont appliquées à construire cette confiance afin que les employés, à leur tour, fassent confiance naturellement aux successeurs. Cela se révèle un des éléments fondamentaux de l'acceptation. Faire confiance signifierait pour les prédécesseures donner la place aux successeurs en les respectant, leur faire comprendre qu'elles croient en leurs capacités, contribuer à bâtir leur visibilité autant au sein de l'entreprise qu'à l'extérieur, témoigner de la fierté à leur endroit et leur accorder le droit à l'erreur. Pour P1F ${ }^{8}$ et P2F, faire confiance, c'est aussi s'appuyer souvent sur le savoir des successeurs et

4. Troisième entreprise des femmes, interview de la successeure, discours numéro 24.

5. Troisième entreprise des hommes, interview du prédécesseur, discours numéro 81.

6. Successeur de la deuxième entreprise des hommes.

7. Deuxième entreprise des hommes, interview de l'employé $\mathrm{B}$, discours numéro 88.

8. Prédécesseure de la première entreprise des femmes. 
assurer leur promotion autant dans l'entreprise qu'auprès des fournisseurs et des associations. Selon leurs dires, cette manière de procéder a renforcé l'estime de soi et la confiance du successeur tout en lui permettant de gagner l'acceptation de ceux qu'il aurait à diriger. Selon leurs dires: "Même si l'on fait des erreurs, ce n'est pas grave, [...] Parce que si l'on a la confiance de la patronne, ça ne veut pas dire que les autres vont avoir confiance en vous» (E4F-Int-Succ, 71). «Elle lui a fait confiance et beaucoup. [...] et elle le consulte beaucoup et le respecte [...] Ça, là, c'est beaucoup pour inviter les employés à accepter quelqu'un» (E2F-Int-Empl-A, 101).

Protéger le successeur. Les énoncés de tous les répondants interviewés nous autorisent à penser que les quatre prédécesseures comprenaient une chose: protéger les successeurs permettait à ces derniers de commettre moins d'erreurs et de se faire accepter sans trop de difficultés. Ainsi, dans le processus du transfert de la philosophie de vie, de la philosophie de gestion et des responsabilités (Hugron, 1993, Lajeunesse, 1989), toutes les quatre ont créé des occasions qui, aux dires même des successeurs, ont consolidé leur acceptation. Elles les ont protégés de toute résistance, de l'insécurité, des adversités et des dommages possibles venant des autres membres de l'entreprise. Elles ont aussi scruté l'environnement afin de déterminer les informations utiles aux successeurs et les ont épaulés dans la mise en œuvre des projets spéciaux qu'ils avaient à réaliser. De plus, le fait que presque toutes ont procédé à la préparation des employés à l'éventualité de la mise en place des successeurs, ce qui se serait fait en douceur, sans les avoir imposés de façon cavalière, aurait été un élément déterminant pour l'acceptation: «Elle m'a beaucoup protégée aussi au début contre l'associé et les employés» (E3F-Int-Succ, $\left.61^{9}\right)$. «Alors ça s'est officialisé en douceur [...] J'ai procédé ainsi pour les protéger» (E4F-Int-Préd, $\left.56^{10}\right)$. «Tu donnes plus de chance à tes enfants à être acceptés. Tu les protèges plus et tu les fais rentrer en douceur. Ça choque moins» (E3F-Int-Empl-A, 157 $\left.{ }^{11}\right)$.

Adopter un style de leadership «maternaliste». P1F, P2F et $\mathrm{P} 3 \mathrm{~F}$ ont fait montre d'une présence soutenue auprès de leurs successeurs. Elles se sont employées à évaluer le contexte émotionnel dans lequel les successeurs se trouvent et les ont amenés à prendre de meilleures décisions. Aux dires d'une employée et d'un successeur: «Je trouve que c'est parce que madame Guimond est plus humanitaire, plus maman, qu'elle sait comment présenter

9. Troisième entreprise des femmes, interview du successeur, discours numéro 61.

10. Quatrième entreprise des femmes, interview du prédécesseur, discours numéro 56.

11. Troisième entreprise des femmes, interview de l'employé A, discours numéro 157. 
son fils, qu'elle le fait accepter bien par nous autres» (E2F-Int-Empl-A, 47). «Une maman qui tient compte comme cela des sentiments de son fils a du mérite, car elle m'a aidée à me faire pardonner» (E4F-Int-Succ, 48).

Mettre l'accent sur le travail collaboratif. Le travail collaboratif fait partie intégrante des valeurs fondamentales de l'entreprise de P1F, P2F et $\mathrm{P} 4 \mathrm{~F}$. Les prédécesseures reconnaissent l'importance de s'assurer que leurs successeurs collaborent avec les employés de manière interdépendante tout en mettant l'accent sur la cohésion d'équipe. Le fait d'amener le successeur à travailler en équipe aurait facilité l'acceptation de ceux-ci : «Ce qu'ils aiment beaucoup, c'est l'esprit de famille qu'on réussit à faire vivre, et puis la possibilité de venir nous parler en tout temps, puis ils savent qu'ils vont être écoutés [...] et ça facilite l'acceptation» (E3F-Int-Succ, 83). «Moi, j'aime l'ambiance d'équipe et la façon de dire que nous réussissons tous ensemble» (E2F-Int-Empl-B, 59).

\subsubsection{Quels comportements pour les hommes chefs d'entreprise?}

Inspirer le successeur. Pour un prédécesseur qui a bâti une entreprise gagnante, il est important que le successeur le perçoive comme un modèle à suivre. C'est le prédécesseur qui donne le ton et décide des rudiments des métiers. Il est perçu comme une source d'inspiration pour le successeur, car c'est lui qui porte la vision de l'entreprise. Voici ce que les successeurs nous disent: "Mon père m'a communiqué sa vision de faire du bon travail, même si je n'aime pas sa façon d'être dur avec nous autres. Je dois faire comme lui» (E1H-Int-Succ, 28). "Ce qui rend facile, c'est que, Andy et moi, on a la même vision au niveau de l'entreprise, il n'y a pas d'autre modèle à suivre» (E2H-IntSucc, 106). «[...] Il est son modèle» (E3H-Int-Empl-B, 198).

Amener le successeur à faire ses preuves. Presque tous les chefs d'entreprise ont exigé de leurs successeurs des rites de passage avant leur acceptation définitive. Pour franchir ces étapes, les successeurs doivent faire leurs preuves par rapport à l'intérêt qu'ils accordent à l'entreprise, à leur engagement et investissement dans l'entreprise. Ces preuves relatives à toute démonstration d'aptitude et de compétences de la part des successeurs dans la réalisation des tâches qui leur sont dévolues seraient considérées comme des épreuves ou rites d'initiation auxquels les successeurs doivent se soumettre et qu'ils doivent réussir. Voici ce que pensent les répondants: «Et je pense que j'avais fait mes preuves et qu'il pouvait me faire confiance lorsqu'il était parti, et cela a été bon pour mon acceptation» (E1H-Int-Succ, 19). «Elle a fait ses preuves, oh oui qu'elle les a faites!» (E3H-Int-Empl,126). Dans le but de prouver aux 
autres leur intérêt et leur degré d'investissement dans l'entreprise, il arrive aussi que $\mathrm{S} 1 \mathrm{~F}^{12}$ et $\mathrm{S} 3 \mathrm{~F}$ travaillent souvent en fin de semaine, et parfois très tard le soir, pour régler certains dossiers prioritaires.

Mettre l'accent sur le travail dyadique. Là où les femmes semblent mettre l'accent sur le travail collaboratif, les hommes semblent préférer les rencontres dyadiques. Au lieu de faire travailler le successeur et les employés ensemble, les prédécesseurs organisent souvent des rencontres dyadiques au cours desquelles ils discutent à propos des objectifs fixés, de la marche à suivre et des difficultés rencontrées. "Quand je fais des erreurs [...], je le croise personnellement dans son bureau, puis dans mon bureau puis on va en discuter, et voir pourquoi les problèmes se reproduisent» (E3H-Int-Succ, 191). "Les rencontres se font à deux, soit avec mon père ou avec les coachs avant d'aller aux employés» (E2H-Int-Succ, 146). «On se rencontre souvent à deux, car il n'aime pas perdre la face devant les employés » (E3H-Int-Succ, 49).

En somme, il semble, selon les résultats, que les prédécesseures seraient plus enclines à faire accepter leurs successeurs plus rapidement que leurs homologues; en effet, une fois en fonction, contrairement aux hommes, elles leur feraient confiance, les protégeraient et adopteraient, ce que nous avons appelé un leadership «maternaliste» tout en insistant sur le travail collaboratif qui permet au successeur de tisser des liens avec les membres de l'entreprise. Cependant, pour les hommes, une différence est notoire: non seulement les successeurs doivent passer par les rites de passage, ils sont aussi amenés à les prendre comme des modèles à suivre, alors que pour les femmes, cela devait se faire naturellement. Maintenant on peut se demander quels seraient les déterminants de l'acceptation pour les répondants.

\subsection{Analyse comparative des déterminants de l'acceptation du successeur selon les répondants des PME dirigées par les femmes et les hommes}

L'entrée du successeur dans l'entreprise commandait alors que celui-ci soit accepté par les membres dont l'accord ou le consentement à travailler avec le successeur était primordial. Selon les analyses intra-cas des femmes et des hommes chefs d'entreprises, il y aurait acceptation du successeur $a$ ) lorsque les prédécesseurs mettent en place leurs successeurs, leur permettant de gravir les échelons en douceur, $b$ ) leur permettant l'acquisition des savoirs et de l'expérience à l'interne, $c$ ) lorsque les valeurs et les comportements des successeurs sont compatibles avec ceux prônés par l'entreprise, et $d$ ) lorsqu'il y a engagement du successeur. Les différences notées résident dans le fait

12. Successeur de la première entreprise des femmes. 
que pour les femmes, la confiance manifestée dès le départ au successeur est primordiale pour une acceptation rapide, alors que pour les hommes, les successeurs doivent non seulement faire leurs preuves dès le départ, mais de plus faire montre d'intérêt, d'initiative et d'investissement.

Gravir les échelons en douceur. Le facteur le plus important pour l'acceptation du successeur est la manière dont les successeurs ont gravi les échelons. Les répondants ont reconnu de façon unanime qu'il fallait procéder à la mise en place du successeur, sans l'imposer, sans rien brusquer, avec patience, sans brûler d'étapes; autrement dit, gravir les échelons en douceur afin que les employés apprivoisent le changement. P1F, P2F et P4F s'y sont pris en "glissant des petits mots», en faisant comprendre aux employés de se référer aux successeurs pour les tâches les concernant. Nous remarquons que presque tous les successeurs ont suivi un cheminement similaire.

Acquérir des savoirs et de l'expérience à l'interne. Les successeurs font leur entrée dans les fonctions qui forment la base de l'organisation. Ceux-ci se familiarisent donc avec chacun des services, donc avec les employés, en commençant au bas de l'échelle et en y remplissant des tâches précises pour accéder graduellement aux échelons supérieurs avant le règne conjoint: «Elle a fait tous les échelons au niveau du SPA et elle s'est fait connaître. Je glissais des petits mots» (E1F-Int-Préd, 24). «[...] Elle n'a pas imposé d’un coup Ignace. Ça s'est fait graduellement. On accepte mieux ça» (E2F-Int-Empl-A, 29).

Dans le cas des hommes, la particularité est que les successeurs devaient gravir les échelons, pas à n'importe quel prix, mais en faisant leurs preuves et en montrant clairement leur degré d'intérêt, d'initiative, d'engagement et d'investissement. Pour $\mathrm{P} 1 \mathrm{H}^{13}$, il faut que $\mathrm{S} 1 \mathrm{H}$ lave d'abord les planchers pour se faire accepter: «[...] Mais, Monique est plus docile. Pour se faire accepter, je lui ai dit qu'elle doit laver les planchers devant les employés, commencer en bas. Moi, je l'ai fait. Il faut travailler fort pour leur montrer que tu es forte» (E1H-Int-Préd, 32014).

Les valeurs et les comportements des successeurs sont congruents. De plus, au cours du processus d'acceptation, les successeurs impliqués dans l'entreprise dès leur jeune âge ont eu à apprendre, autant de leurs parents que des employés, les rouages, les valeurs, les croyances et la vision de l'entreprise, et à adopter des comportements qui répondent aux attentes des membres (Barach et al., 1988). Ils doivent alors prouver hors de tout doute qu'ils adhèrent à la culture de l'entreprise. Selon les répondants:

13. Prédécesseur de la première entreprise des hommes chefs d'entreprise.

14. Première entreprise des hommes chefs d'entreprise, interview du prédécesseur, discours numéro 320. 
«Ce qu'elle a fait, c'est qu'elle remontait toujours aux valeurs fondamentales et aux objectifs qu'on poursuivait» (E3F-Int-Succ, 61). «Fidel a les mêmes valeurs que l'entreprise et c'est bon pour l'acceptation» (E3F-Int-Empl-A, 109). Selon $\mathrm{S} 2 \mathrm{H}$ : «Il l'a bien éduquée, il a communiqué les bonnes valeurs, ce qui fait que moi, je n'ai pas de problème avec ça» (E3H-Int-Empl-B, 65). «Elle, elle retrousse ses manches, elle n'a pas peur de se salir les mains. À mes yeux, c'est ça une patronne de bonne valeur. Et c'est facile pour nous de travailler avec elle» (E1H-Int-Empl-A, 147).

Le successeur est engagé. Partageant la même conviction que $\mathrm{P} 1 \mathrm{H}, \mathrm{P} 2 \mathrm{H}$ et $\mathrm{P} 3 \mathrm{H}$ ont tout mis en œuvre afin que leurs successeurs prouvent leur engagement. C'est à partir de ces preuves que l'acceptation des prédécesseurs est accordée afin que les employés, à leur tour, acceptent les successeurs. Selon un employé: «[...] ça s'est fait naturellement. Pour le père, il faut qu'elle prouve son engagement, mais là, il reste du chemin à faire encore » (E2H-Int-Empl-B, 184). Un employé de Jetpur dit à ce propos: «Premièrement Caroline, il l'intégrait lentement mais sûrement, mais il faut qu'elle montre de quoi elle est capable encore [...] nous autres, on l'accepte de même» (E3H-Int-Empl-B, 63).

\section{Discussion et conclusion}

La transmission des entreprises restant un champ peu exploité, cette recherche avait pour principal objectif de faire un pas supplémentaire en matière de conceptualisation des comportements découlant des styles d'accompagnement adoptés par les femmes et les hommes chefs d'entreprise en situation de retrait pour l'acceptation de leurs successeurs. Elle a atteint quelque peu son objectif, car elle a permis de décrire, de comprendre et de comparer les comportements qu'adoptent les femmes et les hommes chefs d'entreprise pour favoriser l'acceptation de leurs successeurs auprès du personnel de l'entreprise, ce qui n'a jamais été fait auparavant. La compréhension des comportements des prédécesseurs pour l'acceptation des successeurs nous a amenés à tenir compte et de la théorie relationnelle, qui, selon Buttner (2001), Gilligan (1986) et Miller (1991), peut servir de cadre de référence pour l'étude du comportement des femmes et des hommes entrepreneurs, et de la théorie du leadership transformationnel et transactionnel (Bass et Avolio, 1994). Comme mentionnée dans les pages précédentes, l'acceptation du successeur s'est révélée dans notre étude un élément très important pour la pérennité de l'entreprise. Sa concrétisation a donc nécessité des comportements propres aux hommes et des comportements propres aux femmes, ce qui ne corrobore pas nécessairement l'idée des auteurs qui soutiennent que le cadre relationnel peut s'appliquer autant aux hommes qu'aux femmes. 
Premièrement, sur le plan théorique, nos résultats apportent un élément particulier non révélé dans les écrits, à savoir que les femmes et les hommes n'ont pas la même façon de concevoir l'acceptation de leurs successeurs. Le processus pour les femmes se passe alors de façon continue (voir la figure 2) puisque le fait d'établir de bonnes relations avec les successeurs et de leur faire confiance a amené automatiquement les employés à faire confiance aux successeurs et à les accepter.

FIGURE 2

Modèle linéaire d'acceptation du successeur par les femmes prédécesseures

Relation
positive $\rightarrow \begin{aligned} & \text { Choix du } \\ & \text { sucesseur }\end{aligned} \rightarrow$ Confiance $\rightarrow \begin{gathered}\text { Octroi } \\ \text { acceptation }\end{gathered} \rightarrow \begin{gathered}\text { Octroi } \\ \text { acceptation }\end{gathered}$

Cela nous amène à parler du phénomène de la contagion sociale du chef d'entreprise aux employés, puisque selon la théorie de la contagion sociale, les membres d'un groupe social ont tendance à adapter leurs comportements à ceux des autres membres du même groupe (Friedkin, 2001; Leenders, 2002), à plus forte raison qu'il s'agit dans notre cas de transfert de la direction d'une entreprise par une prédécesseure créatrice et dirigeante de son entreprise à son successeur. Non seulement la confiance, dans notre étude, semble constituer la fondation de l'acceptation, mais en outre elle semble être un gage de succès des transmissions permettant la concrétisation rapide de l'acceptation. Pourquoi les femmes n'ont-elles éprouvé aucune difficulté à faire confiance à leurs successeurs? Est-ce parce que, comme l'ont souligné Cadieux et al. (2002) dans leur étude sur la succession dans les entreprises familiales dirigées par les femmes, les rapports entre les mères entrepreneures et leurs enfants basés sur l'empathie seraient habituellement différents des relations entre les pères entrepreneurs et leurs enfants basés sur le contrôle et les preuves? De plus, serait-ce parce que les mères connaîtraient mieux leurs enfants, leurs capacités et leur potentiel, puisque, selon Salganicoff (1990), l'éducation des enfants incombant de façon générale aux mères et non aux pères, il serait plus probable qu'elles puissent leur faire confiance sans se poser de questions?

Concernant les hommes, le processus semble prendre plus de temps et se fait plutôt en escalier, en deux temps. Les prédécesseurs, de façon générale, ayant plus de difficulté à faire confiance à leurs successeurs, garçon comme fille, auraient tendance, comme l'a mentionné Lajeunesse (1989), à tester leurs compétences en les mettant dans des conditions de preuves afin de montrer d'abord à eux-mêmes, ensuite aux employés, ce dont ils sont capables. Cela semble correspondre au style de gestion par exception active de la théorie 
du leadership transactionnel (Bass et Avolio, 1994). C'est aussi à partir du moment où les preuves sont faites par les successeurs que la confiance des prédécesseurs s'installe, qu'ils peuvent accepter leurs successeurs, amenant ainsi les employés à faire comme eux (voir la figure 3 ).

\section{FIGURE 3}

Modèle en escalier en deux étapes du processus d'acceptation du successeur par les hommes prédécesseurs

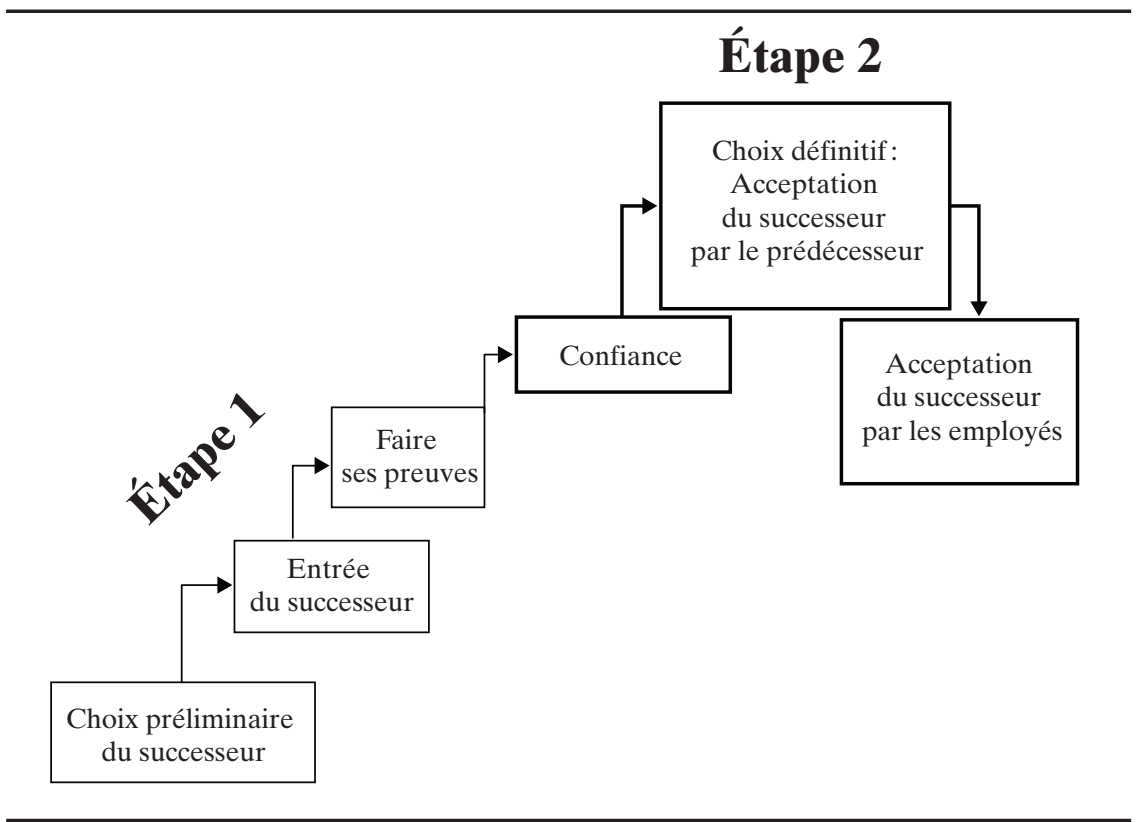

D'ailleurs, l'étude faite par Lorrain, Powers et Koffi (2005) relève les difficultés que les prédécesseurs masculins éprouvent à faire confiance et à assurer correctement leur relève. Les successeurs se sont plaints, lorsque rencontrés par les chercheurs, du contrôle exagéré et du manque de confiance de la part de leurs pères qui ont de la difficulté à tenir compte des émotions et frustrations (Guionnet et Neveu, 2004) de leurs successeurs. Ces résultats qui semblent conclure à une différence fondamentale entre la manière de procéder des hommes et celle des femmes portent à réflexion. N'est-il pas permis d'affirmer que le cadre relationnel, tel que le présente Buttner (2001), ne s'applique pas vraiment aux hommes et que les prédécesseurs semblent adopter un style de gestion qui se résume à «diriger-évaluer-faire confiancereconnaître» et les prédécesseures, un style de gestion consistant à «faire confiance-reconnaître-diriger-évaluer»? 
Deuxièmement, nos résultats ont mis au jour des dimensions nouvelles relativement au cadre relationnel (Buttner, 2001), ne serait-ce qu'à propos des prédécesseures. Non seulement les femmes ont manifesté une habileté à protéger et à épauler leur progéniture, ce qui va en partie dans le même sens que Buttner (2001), mais aussi elles ont mis l'accent, non pas sur le travail d'équipe, mais plutôt sur le travail collaboratif qui met en exergue la collaboration et l'interdépendance professionnelle et sociale. De plus, il serait possible d'affirmer que le comportement de leadership «maternaliste», nouvelle dimension, particulier aux femmes, corrobore l'idée de certains auteurs relevant que les femmes, de façon générale, posséderaient certains atouts leur permettant d'adopter un style de leadership transformationnel (Bass et Avolio, 1994; Eagly et al., 2003; Riebe, 2005; Rosener, 1990) axé sur les relations interpersonnelles et sur le développement des personnes qui les côtoient (Buttner, 2001; Chodorow, 1978; Gilligan, 1986; Miller, 1991). Avec de tels résultats, qui nous amènent à relever une différence dans les comportements adoptés par les prédécesseurs hommes et femmes, ne nous est-il pas permis de penser qu'il existerait chez certaines prédécesseures un modèle dit féminin (Alimo-Metcalfe, 2007; Avolio et Bass, 2002; Eagly et al., 2003; Rosener, 1990) qui découlerait d'un style dit «féminin» relationnel, collaboratif et interactif proposé par le cadre relationnel (Buttner, 2001; Fletcher, 1998; Miller, 1991) et transformationnel (Bass et Avolio, 1994) et différent d'un modèle dit «masculin»? Dans l'affirmative, serait-ce une question de prédisposition, de maternité, comme le supposent Gilligan (1986), Miller (1991) et Buttner (2001), ou une question d'apprentissage social comme le souligneraient certains auteurs (Ahl,2006; Ely et Padavich,2007; Guionnet et Neveu, 2004; Héritier, 2005) ? Justement, pour ces derniers, d'un côté, certains attributs «masculins», tels que la prédisposition au pouvoir et à la pratique de la censure émotionnelle, et certains attributs «féminins», comme la prédisposition à se mettre en relation avec les autres, n'emprisonnent-ils pas les hommes et les femmes dans des modèles masculin et féminin très normatifs?

Troisièmement, concernant les déterminants de l'acceptation, Barach et al. (1988) et Sathe (1985) soutiennent que le successeur est accepté non seulement en fonction de la manière dont il fait son entrée dans l'entreprise, mais aussi en fonction du degré de confiance et du respect qui lui sont accordés. Nos résultats corroborent en partie ces assertions. Si, selon certains auteurs (Hugron, 1993; Lajeunesse, 1989; Richer et al., 2004), une arrivée précipitée ou imposée à la direction par le prédécesseur ou le successeur peut être une source de difficultés qui ferait échouer le processus, nos résultats indiquent clairement que la mise en place de la relève en douceur est une des plus importantes stratégies favorisant l'acceptation du successeur. Faire entrer les successeurs, notamment par le bas de l'échelle, leur a donc permis de se familiariser avec chacun des services, de socialiser avec les autres membres 
de l'entreprise, de gravir graduellement les échelons et de rapidement se faire accepter. Une contribution nouvelle dans cette étude est d'avoir trouvé qu'il ne suffit pas de faire son entrée de préférence par «le bas de l'échelle», mais qu'il faut encore user de stratégies particulières, comme les femmes ont su si bien le faire, afin que l'acceptation du successeur soit favorisée, sinon assurée.

Sur le plan scientifique, aborder la problématique de la succession sous l'angle de l'entrepreneuriat féminin est en soi une avancée, en raison de la rareté des études qui s'y sont intéressées. De plus, les écrits décrivant les qualités des femmes entrepreneures versent beaucoup plus dans la généralité en présentant celles-ci comme collaboratives, participatives, transformationnelles, sans toutefois proposer de supports théoriques et empiriques valides. Dans une modeste mesure, notre étude est parvenue à le faire, car les comportements adoptés par les prédécesseurs pour faciliter l'acceptation de leurs successeurs semblent avoir porté des fruits, outre de bien cadrer avec le genre féminin.

D'un point de vue managérial, le phénomène de la contagion sociale au regard de la relation tissée et de la confiance est fondamental pour la réussite de la transmission (Koffi et Lorrain, 2011). Notre étude pourrait aider les prédécesseurs à améliorer leur approche relationnelle par rapport à leurs successeurs et à leur faire confiance. Les conseillers qui interviennent auprès des chefs d'entreprise pourraient adapter leurs discours, leurs approches et leurs outils d'intervention aux prédécesseurs. En sachant que les prédécesseures semblent adopter un style plus «maternaliste», plus féminin, les prédécesseurs pourraient-ils faire de même? Les conseillers pourraient organiser à cette fin des ateliers de sensibilisation et d'interventions axés sur les comportements gagnants dits féminins, se servir des cadres de référence appropriés; sinon, ils pourraient avoir des problèmes avec les prédécesseurs féminins qui ne se reconnaîtraient pas dans les modèles masculins qui sont encore communément utilisés.

Forts des études consultées, nous pensons que les résultats obtenus ont pu apporter certains éléments de réponse. La quête de réponses plus soutenues nous mènera vers une démarche méthodologique quantitative basée sur un échantillon plus large qui nous permettra de relever et de caractériser, sous forme d'un modèle théorique robuste, un ensemble de stratégies d'acceptation dont les femmes et les hommes en processus de succession se serviront afin de mieux assurer la continuité de leurs entreprises.

La présente recherche comporte des limites dont la principale découle de la stratégie de recherche. Les sept entreprises que nous avons retenues correspondent toutes aux critères généralement attribués aux PME. Or, comme le souligne Santiago (2000), la taille des entreprises a une influence 
sur le processus de succession. Quels seraient donc les types de comportement de leadership que les prédécesseurs des grandes entreprises devraient adopter pour favoriser l'acceptation des successeurs et même des repreneurs, en l'absence de successeurs? Allant dans le même sens, Cadieux et al. (2002) et Richer et St-Cyr (2001) mentionnent que les conjoints ont un ou plusieurs rôles à jouer au cours du processus de succession. Quels comportements devraient-ils adopter en vue de l'acceptation de leur successeur? Lorsqu'il sera question de repreneurs, devraient-ils intervenir? Et de quelle manière? Ce sont des éléments que nous n'avons pas abordés et qui auraient pu mieux éclairer le processus de succession. Ici, non plus, nous n'avons pas toutes les réponses. Enfin, l'échantillon par choix raisonné de sept cas d'entreprises, quatre cas de femmes et trois cas d'hommes, est loin d'être représentatif de l'ensemble des situations pour une généralisation sûre, même si selon Yin (1994), pour une étude de cas comparative, quatre à dix cas suffisent. Aussi serait-il intéressant de poursuivre la recherche en considérant un plus grand nombre de cas.

\section{Bibliographie}

AHL, H. (2006), «Why research on women entrepreneurs need new directions », Entrepreneurship Theory and Practice, vol. 30, $\mathrm{n}^{\circ}$ 5, p. 595-623.

Alimo-MetCAlFE, B. (2007), «Les sexes et le leadership: un plafond de verre ou de béton armé ?», Téléscope, vol. 13, nº 4, p.14-41.

ARDTS, J., P. JANSEN et M. VAN DER VELDE (2001), «The breaking in of new employees: effectiveness of socialisation tactics and personnel instruments », The Journal of Management Development, vol. 20, nº 2, p. 159-163.

ASHFORTH, B.E., A.M. SAKS et R.T. LEE (1998), «Socialisation and newcomer adjustement: the role of organizational context», Human Relations, vol. 51, $\mathrm{n}^{\mathrm{o}}$ 7, p. 897-917.

ASTRACHAN, J.H. et M.C. SHANKER (2003), «Contribution to the U.S. economy: a closer look», Family Business Review, vol. 3, p. 211-219.

AVOLIO, B.J. et B.M. BASS (2002), Manual for the Multifactor Leadership Questionnaire (Form $5 X$ ), Redwood City, CA, Mindgarden.

BARACH, J.A., J. GANITSKY, J.A. CARSON et B.A. DOOCHIN (1988), «Entry of the next generation: strategic challenge for family business », Journal of Small Business Management, vol. 26, $\mathrm{n}^{\mathrm{O}}$ 2, p. 49-56.

BARbot , M.-C. et K. Richome-HuET (2007), «Pilotage de la reprise et de la succession dans le cas des entreprises artisanales», Économies et Sociétés, vol 16, no 1, p. 57-90.

BARRETT, M. et K. MOORES (2009), «Spotlights and shadows: preliminary findings about experiences of women in family business leadership », Journal of Management and Organization Studies, vol. 15, n 3, p. 363-377. 
BASS, B.M. et B.T. AVOLIO (1994), «Shatter the glass ceiling: women may make better managers », Human Resources Management, vol. 33, nº 4, p. 549-560.

BAYAD, M. et M.-C. BARBOT (2002), «Proposition d'un modèle de succession dans les PME familiales: étude de cas exploratoire de la relation père-fille», dans Actes du sixième congrès international francophone sur la PME, Montréal, du 30 octobre au $1^{\mathrm{er}}$ novembre.

BRUCE, D et D. PICARD (2005), «La relève, la clé de la réussite: la relève des PME et la prospérité économique au Canada », Référence du 26 avril 2007 de : <http :// www.fcei.ca/researchf/reports/rr3007f.pdf $>$.

BRUIN (De) A., C. BRUSH et F. WELTER (2007), «Advancing a framework of coherent research on womens'entrepreneurship », Entrepreneurship Theory and Practice, vol. 31, no 3, p. 323-340.

BRUSH, C. (1992), «Research on women business owners : past trends, a new perspective and future directions », Entrepreneurship Theory and Practice, vol. 16, $\mathrm{n}^{\circ} 4$, p. 5-30.

BUTLER, J. (1990), Gender Trouble: Feminism and the Subversion of Identity, Londres, Routledge.

BUTTNER, E.H. (2001), «Examining female entrepreneurs'management style: an application of a relational frame», Journal of Business Ethics, vol. 29, $\mathrm{n}^{\circ} 3$, p. 253-269.

CABRERA-SUÁREZ, K. (2005), «Leadership transfer and the successor's development in the family firm », Leadership Quarterly, vol. 16, nº 1, p. 71-83.

CADIEUX, L. et F. BROUARD (2009), La transmission des PME: perspectives et enjeux, Québec, Presses de l'Université du Québec.

CADIEUX, L., J. LORRAIN et P. HUGRON (2002), «La succession dans les entreprises familiales dirigées par les femmes: une problématique en quête de chercheurs », Revue internationale PME, vol. 15, $\mathrm{n}^{\mathrm{o}}$ 1, p. 115-130.

CHOdorow, N. (1978), The Reproduction of Mothering, Bekerley, CA, University of California Press.

COLE, P. (1997), «Women in family business », Family Business Review, vol. 10, n 4, p. 353-371.

D'ANDRIA A. et M.-C. CHALUS-SAUVANNET (2007), «De l'entrée à la socialisation organisationnelle du repreneur : réalités et difficultés. Le cas d'un établissement hôtelier ", Économie et sociétés, vol. 41, nº 1, p. 121-143.

DESCHENAUX, F. et S. BOURDON (2005), «Introduction à l'analyse qualitative informatisée à l'aide du logiciel QSR Nvivo 2.0.», Les cahiers pédagogiques de l'Association pour la recherche qualitative, Sherbrooke, Québec.

DUCHÉNEAUT, B. (1997), «Les femmes entrepreneurs à la tête de PME», Rapport réalisé pour la Conférence de l'OCDE. Les femmes entrepreneurs à la tête de PME: une nouvelle force pour l'innovation et la création d'emplois, Paris, $16-18$ avril. 
EAGLY, A.H., M.-C. JOHANNESEN-SCHMIDT et M.L. VAN ENGEN (2003), «Transformational, transactional, and laissez-faire leadership styles: a meta-analysis comparing women and men », Psychological Bulletin, vol. 129, nº 4, p. 569-591.

ELY, R. et I. PADAVICH (2007), «A feminist analysis of organizational research on sex differences », Academy of Management Review, vol. 32, n 4, p. 1121-1143.

FIEGENER, M.K., B.M. BROWN, R. ALL. PRINCE et K.M. FILE (1996), «Passing on strategic vision: favoured modes of successor preparation by CEOS of family and nonfamily firms », Journal of Small Business Management, vol. 34, n 3, p. $15-26$.

FISHER, E., W. REUBER et L. DYKE (1993), «A theoretical overview and extension of research on sex, gender and entrepreneurship », Journal of Business Venturing, vol. 8, p. 151-168.

FLETCHER, J. (1998), «Relational practice : a feminist reconstruction of work», Journal of Management Inquiry, vol. 7, $\mathrm{n}^{\circ}$ 2, p. 163-186.

FONDAS, N. (1997), «Feminization unveiled:management qualities in contemporary writings », Academy of Management Review, vol. 22, nº 1, p. 257-282.

FOSCHI, M. (2000), «Double standards for competence: theory and research», Annual Review of Sociology, vol. 26, p. 21-42.

FRIEDKIN, N. E. (2001), «Norm formation in social influence networks», Social Networks, vol. 23, p. 167-189.

GiLligAn, C. (1986), Une si grande différence, Paris, Flammarion.

Guionnet, C. et E. Neveu (2004), Féminin/Masculin. Sociologie du genre, Paris, Armand Colin.

GRENIER, C. et E. JOSSERAND (1999), «Recherche sur le contenu et recherche sur le processus », dans R.-A. Thiétart (dir.), Méthodes de recherche en management, Paris, Dunod, p. 104-136.

HADDADJ, S. et A. D'ANDRIA (2001), «Mesurer les phénomènes agissant sur la transmission des PME familiales », Revue française de gestion, vol.132, p. 11-22.

HANDLER, W.C. (1990), «Succession in family firms: a mutual role adjustment between entrepreneur and next-generation family members », Entrepreneurship Theory and Practice, vol. 15, $\mathrm{n}^{\mathrm{o}}$ 1, p. 37-51.

HELGESEN, S. (1990). The Female Advantage:Women's Ways of Leadership, $1^{\text {re }}$ édition, New York, Doubleday Currency.

HÉRITIER, F. (2005), Hommes, femmes, la construction de la différence, Paris, Le Pommier, Cité des sciences et de l'industrie.

HISRICH, R. et C. BRUSH (1984), «The women entrepreneur: management skills and business problems », Journal of Small Business Management vol. 22, $\mathrm{n}^{\circ}$ 1, p. 30-37.

HLADY-RISPAL, M. (2002), La méthode des cas: application à la recherche en gestion, Bruxelles, De Boeck Université.

HOLLANDER, E. (1992), «The essential independence of leadership and followership », Current Directions in Psychological Science, vol. 1, $\mathrm{n}^{\circ}$ 2, p. 71-75. 
Hugron, P. (1993), «L'entrepreneur et la succession d'entreprise: analyse de douze processus (1985)», Cahier de recherche sur les entreprises familiales, n ${ }^{\circ}$ GREF-93-03, École des Hautes Études commerciales, Montréal, Québec.

JOLICEUUR, A. (1994), La succession dans les entreprises familiales: un processus de communication, mémoire de maîtrise, Montréal, Université de Montréal.

KOFFI, V. (2008), L'intégration du successeur dans les PME familiales: une étude de cas comparative des stratégies des prédécesseures et des prédécesseurs, thèse de doctorat inédite, Trois-Rivières, Université du Québec à Trois-Rivières.

KOFFI, V. et J. LORRAIN (2011), "Comment des femmes à la tête de PME réussissentelles leur succession?», Revue internationale de gestion, vol. 36, nº 1, p. 35-40.

LAJEUNESSE, C. (1989), La succession managériale dans la PME familiale: études de règne-conjoint, mémoire de maîtrise, Montréal, École des Hautes Études commerciales.

LANSBERG, I. et J.H. ASTRACHAN (1994), «Influence of family relationships on succession planning and training: the importance of mediating factors », Family Business Review, vol. 7, no 1, p. 39-59.

LEENDERS, R.T.A.J. (2002), «Modeling social influence through network autocorrelation constructing the weight matrix», Social Networks, vol. 24, nº 1, p. 21-47.

LONGENECKER, C.O. et N. NYKODYM (1996), «Public sector performance appraisal effectiveness: a case study», Public Personnel Management, vol. 25, $\mathrm{n}^{\circ} 2$, p. 151-164.

LORRAIN, J., T. POWERS et V. KOFFI (2005), La relève entrepreneuriale dans les entreprises: étude de trois régions du Québec, rapport inédit, Ministère du Développement économique, Innovation et Exportation.

Miller, J.B. (1986), Toward a New Psychology of Women, $2^{\mathrm{e}}$ éd., Boston, MA, Beacon Press.

MiLleR, J.B. (1991), «The development of women's sense of self», dans Jordan, A. Kaplan, J.B. Miller, I.P. Stiver et J. Surrey (dir.), Women's Growth in Connection, New York, Guilford Press, p. 11-26.

MuRPHY, K.R. et J.N. CleVElAnd (1995), Understanding Performance Appraisal: Social Organizational and Goal-based Perspectives, Thousand Oaks, CA, Sage Publications.

PAillé, P. et A. MUCCHIELli (2003), L'analyse qualitative en sciences humaines et sociales, Paris, Armand Colin.

POUDRIER, L. (2000), L'acceptation d'un système d'appréciation du rendement organisationnel: une étude longitudinale, thèse de doctorat, Montréal, Québec, Université de Montréal.

POWELL, G.N. (1990), «One more time: do female and male managers differ?» Academy of Management Executive, vol. 4, n 3, p. 68-75.

RAPOPORT, R. et L. BAILYN (1996), Relinking Life and Work: Toward a Better Future, Darby, PA, Diane Publishing, 42 p. 
RICHER, F. et L. ST-CYR (2001), «La planification de la relève dans les PME: statistiques et réflexions », Gestion, revue internationale de gestion, vol. 26, nº 2, p.19-26.

RICHER, F. et L. ST-CYR (2007), L'entrepreneuriat féminin au Québec. Dix études de cas, Montréal, Presses de l'Université de Montréal.

RICHER, F., L. ST-CYR et L. YOUSSEF (2004), «La transmission d'entreprise au Québec: des stratégies diversifiées», Revue internationale de gestion, vol. 29, $\mathrm{n}^{\mathrm{o}} 3$, p. 95-102.

RIEBE, M. (2005), «The leadership style of women entrepreneurs: an alternative model of successful management practices », Actes $d u 50^{e}$ congrès international de l'ICSB, Washington, 16-18 mai.

RoBINSON, S. (2001), «An examination of entrepreneurial motives and their influence on the way rural women small business owners manage their employees», Journal of Developmental Entrepreneurship, vol. 6, $\mathrm{n}^{\circ}$ 2, p. 151-167.

Rosener, J. (1990), «Ways women lead», dans Nancy A. Nichols, Reach for the Top, Boston, MA, Harvard University School Press, p. 13-22.

SALGANICOFF, M. (1990), «Women in family business: challenges and opportunities», Family Business Review, vol. 3, n 2, p. 125-137.

SANTiago, A.L. (2000), «Succession experience in Philippine family business», Family Business Review, vol. 13, n 1, p. 15-40.

SATHE, V. (1985), Culture and Related Corporate Realities, Homewood, IL, Irwin.

SHARMA, P. (2004), «An overview of the field of family business studies: current status and directions for the future », Family Business Review, vol. 17, n 1, p. 1-36.

SONFIELD, M. C. et R.N. LUSSIER (2009), «Gender in family business ownership and management: a six-country analysis », International Journal of Gender and Entrepreneurship, vol. 1, n ${ }^{\circ}$, p. 96-117.

TSUI, A., S.J. PEARCE, L. PORTER et A. TRIPOLI (1997), «Alternative approaches to the employee-organization relationship: does investment in employees pay off? », Academy of Management Journal, vol. 40, p. 1089-1121.

VAN ENGEN, M.L., R. VAN DER LEEDEN et T.M. WiLlEMSEN (2001), «Gender, context and leadership styles: a field study», Journal of Occupational Psychology, vol. 74, p. 581-598.

VECCHIO, R.P. (2003), «In search of gender advantage», The Leadership Quarterly, vol. 14, p. 835-850.

VIKINIAS, T. et G. CARTAN (1993), «Competences of Australian women in management», Women in Management Review, vol. 8, n 3, p. 31-35.

WANOUS, J.P. (1992), Organizational Entry: Recruitment, Selection, and Socialization of Newcomers, Massachusetts, MA, Addison-Wesley.

WANOUS, J.P. et A.E. REICHERS (2000), «New employee orientation program's », Human Resource Management Review, vol. 10, nº 4, p. 17-31.

WEISINGER, H. (1998), Emotional Intelligence at Work, San Francisco, CA, JosseyBass.

YIN, R.K.(1994), Case Study Research:Design and Methods, Applied Social Research Methods Series, 5, $2^{\mathrm{e}}$ édition, Beverley Hills, CA, Sage Publications. 